\title{
Studies on type I collagen in skin fibroblasts cultured from twins with lethal osteogenesis imperfecta
}

\author{
Anna Galicka ${ }^{1}$, Sławomir Wołczyński ${ }^{2}$ and Andrzej Gindzieński ${ }^{1}$ \\ ${ }^{1}$ Department of Medical Chemistry, and ${ }^{2}$ Department of Gynaecological Endocrinology, \\ Medical Academy of Bialystok, Bialystok, Poland
}

Received: 04 March, 2003; revised: 26 May, 2003; accepted: 10 June, 2003

Key words: type I collagen, osteogenesis imperfecta

\begin{abstract}
Studies on type I procollagen produced by skin fibroblasts cultured from twins with lethal type II of osteogenesis imperfecta (OI) showed that biosynthesis of collagen (measured by L-[5- $\left.{ }^{3} \mathrm{H}\right]$ proline incorporation into proteins susceptible to the action of bacterial collagenase) was slightly increased as compared to the control healthy infant. SDS/PAGE showed that the fibroblasts synthesized and secreted only normal type I procollagen. Electrophoretic analysis of collagen chains and $\mathrm{CNBr}$ peptides showed the same pattern of electrophoretic migration as in the controls. The lack of posttranslational overmodification of the collagen molecule suggested a molecular defect near the amino terminus of the collagen helix. Digestion of OI type I collagen with trypsin at $30^{\circ} \mathrm{C}$ for 5 min generated a shorter than normal $\alpha 2$ chain which melted at $36^{\circ} \mathrm{C}$. Direct sequencing of an asymmetric PCR product revealed a heterozygous single nucleotide change $\mathrm{C} \rightarrow \mathrm{G}$ causing a substitution of histidine by aspartic acid in the $\alpha 2$ chain at position 92. Pericellular processing of type I procollagen by the twin's fibroblasts yielded a later appearance of the intermediate $\mathrm{pC}-\alpha 1$ (I) form as compared with control cells.
\end{abstract}

Type I collagen is the major protein in the extracellular matrix of skin, bone, tendons and most other connective tissues. It is synthesized as a soluble procollagen form composed of globular C- and N-propeptides flanking the triple helix. Assembly of the trimer $[\operatorname{pro} \alpha 1(\mathrm{I})]_{2} \operatorname{pro} \alpha 2(\mathrm{I})$ is initiated by the car- boxyl-terminal propeptide domains (McLaughlin \& Bulleid, 1998). The distinctive (Gly-Pro-Hyp) ${ }_{4}$-Gly-Pro-Pro sequence at the C-terminus of the triple helix domain of the $\alpha 1$ and $\alpha 2$ chains is likely to be involved in the formation of a stable triple helix nucleus (Byers, 2001). Following nucleation the triple

\footnotetext{
${ }^{{ }}$To whom correspondence should be addressed: Anna Galicka, Department of Medical Chemistry, Medical Academy of Białystok, 15-230 Białystok 8, Poland; tel.: (48 85) 748 5673; fax: (48 85) 748 5416; e-mail: angajko@amb.ac.bialystok.pl
}

Abbreviation: OI, osteogenesis imperfecta. 
helix conformation is propagated in a zipper-like mechanism from the $\mathrm{C}$ - to the N-terminus (Bachinger et al., 1993). Simultaneously, proline and lysine residues are hydroxylated by specific hydroxylases and hydroxylysine residues are glycosylated by glycosyl transferases. Upon secretion from the cell the propetides are cleaved by $\mathrm{C}$ - and $\mathrm{N}$-proteinases and the collagen monomers are assembled into collagen fibrils.

The triple helix consists of uninterrupted Gly-X-Y triplets in which X and Y can be any amino acid but cysteine or tryptophan. The triplet Gly-Pro-Hyp is the most stabilizing tripeptide sequence and the most frequent, constituting $10 \%$ of all triplets. Both proline and hydroxyproline promote triple helix stability, but a greater stabilizing effect is shown by hydroxyproline (Berg \& Prockop, 1973). Structural abnormalities of type I collagen are the cause of a genetic disorder of connective tissue, osteogenesis imperfecta (OI), characterized by bone fragility and deformity, dentinogenesis imperfecta, alterations in scleral color, hearing loss and shortened body stature. The spectrum of severity of the symptoms of OI (Sillence et al., 1979) is reflected in the heterogeneity of the molecular defects in type I procollagen that cause the disease. Most cases are caused by dominant mutations in COL1A1 or COL1A2, the genes encoding the $\alpha 1(\mathrm{I})$ and $\alpha 2(\mathrm{I})$ subunits of type I collagen, respectively (Kuivaniemi et al., 1991; Prockop, 1992; Gajko-Galicka, 2002). Substitutions of glycine, the smallest amino acid, by other amino acids such as arginine, cysteine, serine or alanine impairs the formation and stability of the collagen triple helix, secretion of procollagen from the cell, extracellular processing and the incorporation of mature collagen into the extracellular matrix (Byers, 1993; Cole, 1997; Galicka et al., 2002).

Studies of type I collagen in fibroblasts cultured from twins with lethal osteogenesis imperfecta were performed. We have found susceptibility of collagen to trypsin digestion and delayed processing of procollagen in OI cells. Although non-glycine substitutions within the triple helix have not been reported to date in osteogenesis imperfecta, we found a heterozygous substitution at the $\mathrm{X}$ position of the Gly-X-Y triplet which might be a factor contributing to the lethal phenotype in the twins.

\section{MATERIALS AND METHODS}

Fibroblast cultures. Skin biopsies from twins with lethal type II of osteogenesis imperfecta and a control (unrelated healthy infant after surgery) were obtained with the approval of the Ethics Committee of the Medical Academy and with informed parental consent. Skin fibroblasts were cultured in Dulbecco's modified Eagle's medium (DMEM) (Gibco, U.S.A.) supplemented with 10\% fetal bovine serum (FBS) (Gibco, U.S.A.), $2 \mathrm{mM}$ glutamine, $50 \mathrm{U} / \mathrm{ml}$ penicillin, $50 \mu \mathrm{g} / \mathrm{ml}$ streptomycin at $37^{\circ} \mathrm{C}$ in $5 \% \mathrm{CO}_{2}$ in an incubator. The cells were used between the 2nd and 8th passages. For the experiments cells were counted in a hemocytometer and inoculated at $2 \times 10^{5}$ cells per well (in 6 well plates, Costar) in $2 \mathrm{ml}$ of growth medium. Cells reached confluence at 2 nd day after inoculation and in most cases such cells were used for the experiments.

Biochemical studies of collagen. Collagen biosynthesis was measured after labeling of confluent fibroblasts for $24 \mathrm{~h}$ with L- $\left[5^{3} \mathrm{H}\right]$ proline $(5 \mu \mathrm{Ci} / \mathrm{ml}, 28 \mathrm{Ci} / \mathrm{mmol}$, Amersham Corp.) in serum-free medium. Incorporation into collagen was determined by digesting the proteins with purified Clostridium histolyticum collagenase (Sigma) according to the method of Peterkofsky et al. (1982). The results are shown as combined values for cell plus medium fractions.

For electrophoretic analysis confluent fibroblast cultures were labeled with $50 \mu \mathrm{Ci} / \mathrm{ml}$ of $\mathrm{L}-\left[5{ }^{3} \mathrm{H}\right]$ proline $(28 \mathrm{Ci} / \mathrm{mmol})$ for $18 \mathrm{~h}$ in serum-free DMEM containing $50 \mu \mathrm{g} / \mathrm{ml}$ of ascorbic acid. Procollagens from the cell layer 
and medium were harvested separately and analysed on a $5 \%$ polyacrylamide gel containing $2 \mathrm{M}$ urea under reducing conditions (Bonadio et al., 1985; Laemmli, 1970). Procollagen was converted to collagen by limited proteolysis $\left(4 \mathrm{~h}\right.$ at $\left.4^{\circ} \mathrm{C}\right)$ with pepsin $(50 \mu \mathrm{g} / \mathrm{ml})$ as described by Bonadio et al. (1985) and electrophoresed on a $5 \%$ gel under nonreducing conditions. The same amount of radioactivity was loaded on each slab (approx. 50000 counts/min). Gels were processed for fluorography with Amplify (Amersham Corp.). Cyanogen bromide treatment of proteins in polyacrylamide gel and separation of the resulting peptides in the second dimension on a $12.5 \%$ polyacrylamide/urea/SDS gel were performed as described by Bonadio et al. (1985).

In order to determine of thermal stability, $\left[{ }^{3} \mathrm{H}\right]$ proline-labeled collagen samples were digested with trypsin $(100 \mu \mathrm{g} / \mathrm{ml})$ for $5 \mathrm{~min}$ at progressively higher temperatures. Digestion products were separated on a 5\% SDS/polyacrylamide gel with $2 \mathrm{M}$ urea under nonreducing conditions (Bonadio et al., 1985; Laemmli, 1970). The thermal denaturation temperature $t_{m}$ was defined as the temperature at which half of the collagen amount was degraded.

Processing of procollagen secreted by fibroblasts was examined by labeling of confluent cells in a 6 well plate with $50 \mu \mathrm{Ci} / \mathrm{ml}$ of $\mathrm{L}-\left[5-{ }^{3} \mathrm{H}\right]$ proline $(28 \mathrm{Ci} / \mathrm{mmol})$ for $24 \mathrm{~h}$, and then replacing the medium with DMEM containing $2 \mathrm{mM}$ nonradioactive proline and 10\% FBS. Media were harvested from individual wells at 24-h intervals over a 3-day period. Procollagen was precipitated from the media and electrophoresed on a $5 \%$ polyacrylamide/urea/SDS gel with dithiothreitol (DTT).

Localization of the molecular defect in COL1A2 gene encoding collagen $\alpha 2(I)$ chain. Total RNA was isolated from cultured skin fibroblasts of the twins and control using Total RNA Prep Plus (A\&A Biotechnology, Gdańsk, Poland) according to the manufacturer's directions. The N-terminal fragment of $\alpha 2(\mathrm{I})$ was amplified by RT-PCR. For firststrand synthesis a reverse primer (Perkin Elmer) located at nt 739-768 of the cDNA sequence and a 15 min incubation with $\mathrm{MuLV}$ reverse transcriptase (Perkin Elmer) at $42^{\circ} \mathrm{C}$ were employed. A forward primer located at nt 189-218 of the cDNA sequence was added for PCR amplification. The conditions of amplification were: one cycle of $1 \mathrm{~min}$ at $94^{\circ} \mathrm{C}, 30$ $\mathrm{s}$ at $70^{\circ} \mathrm{C}$, and $1 \mathrm{~min}$ at $72^{\circ} \mathrm{C}$, followed by $35 \mathrm{cy}$ cles of $30 \mathrm{~s}$ at $94^{\circ} \mathrm{C}, 30 \mathrm{~s}$ at $70^{\circ} \mathrm{C}$, and $45 \mathrm{~s}$ at $72^{\circ} \mathrm{C}$. The reaction product was submitted to agarose gel electrophoresis and column purification (GenElute Agarose spin column, Sigma), and then used for asymmetric amplification with the same reverse primer and REDTaq DNA Polymerase (Sigma) in the conditions described above. The single-stranded PCR product was directly sequenced by the dideoxy chain termination method (Sanger et $a l .$, 1997) using a Sequenase PCR product Sequencing Kit (Amersham). As a sequencing primer the 30-mer 5' CGA CCT GGT GAG AGA GGA GTT GTT GGA CCA 3' (PerkinElmer) located at nt 315-344 was used. Direct sequence analyse of cDNA obtained from two independent RNA preparations were performed in duplicate. As an additional control for the PCR reaction, RNA from control fibroblasts was also amplified and sequenced as described above.

\section{RESULTS}

In skin fibroblasts of twins with lethal form of osteogenesis imperfecta the biosynthesis of collagen (measured by L- $\left[5-{ }^{3} \mathrm{H}\right]$ proline incorporation into proteins susceptible to the action of bacterial collagenase) was slightly increased in comparison with control fibroblasts (Fig. 1). Electrophoretic analysis of type I procollagen isolated from the media and OI fibroblasts showed the same pattern of migration as in controls (Fig. 2A). The structural defect of type I collagen in osteogenesis imperfecta is usually manifested by delayed 
migration of collagen chains in the gel, caused by its overmodification. However, the $\alpha 1$ and $\alpha 2$ chains of type I collagen as well as $\mathrm{CNBr}$ peptides of the collagen chains analyzed by

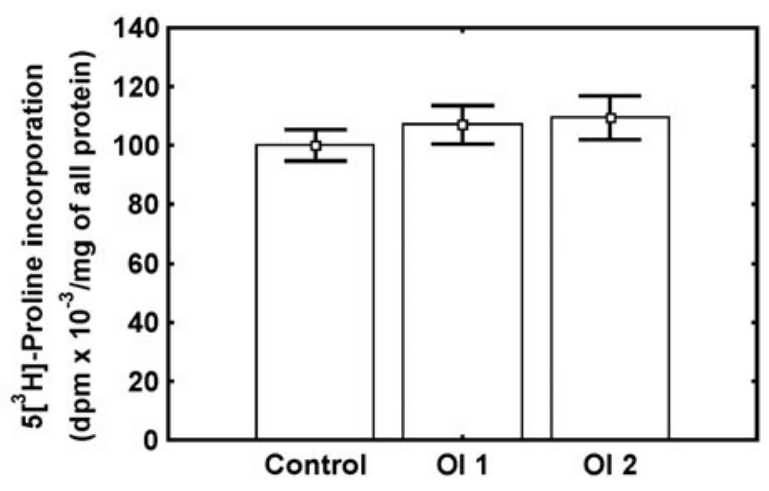

Figure 1. Collagen biosynthesis in control and twins' (OI1, OI2) fibroblasts.

Confluent cells were incubated in serum-free medium for $24 \mathrm{~h}$ with $\left[{ }^{3} \mathrm{H}\right]$ proline. Incorporation into collagen was determined by digesting proteins with collagenase. Each value represents the mean for five independent experiments. The results were submitted to statistical analysis using the Student's $t$-test, accepting $P<$ 0.05 as significant.

SDS/urea/PAGE (Fig. 2B and C, respectively) confirm that OI collagen appears electrophoretically normal. Both procollagen and collagen were well secreted into the medium and there was no evidence of cellular retention. These experimental data point to the presence of a mutation located very close to the amino end of the helical region of either the $\alpha 1(\mathrm{I})$ or $\alpha 2(\mathrm{I})$ chains.

To test for proteolytic susceptibility, OI collagen secreted into the medium was digested with trypsin for $5 \mathrm{~min}$ at increasing temperatures, and the digestion products were separated on a $5 \%$ SDS/urea/polyacrylamide gel (Fig. 3). Digestion performed at 30 or $33^{\circ} \mathrm{C}$ revealed an additional band migrating below the $\alpha 2$ (I) chain. This band was confirmed by the analysis of its $\mathrm{CNBr}$ peptides to be a truncated (amino-terminally shortened) $\alpha 2$ (I) chain (data not shown). Digestion with trypsin at $36^{\circ} \mathrm{C}$ resulted in an almost complete degrada- tion of the shortened $\alpha 2$ (I) protein, whereas normal $\alpha 1(\mathrm{I})$ and $\alpha 2(\mathrm{I})$ remained unaffected up to $39^{\circ} \mathrm{C}$. These results suggest that the trimers of procollagen contained protease-sensitive sequences which, when cleaved, produced $\alpha 2(\mathrm{I})$ chains shorter than normal and protease-stable at $30-33^{\circ} \mathrm{C}$. The melting temperature $t_{\mathrm{m}}$ of OI type III collagen was the same as the control.

On the basis of the biochemical data, the search for the mutation was directed to the

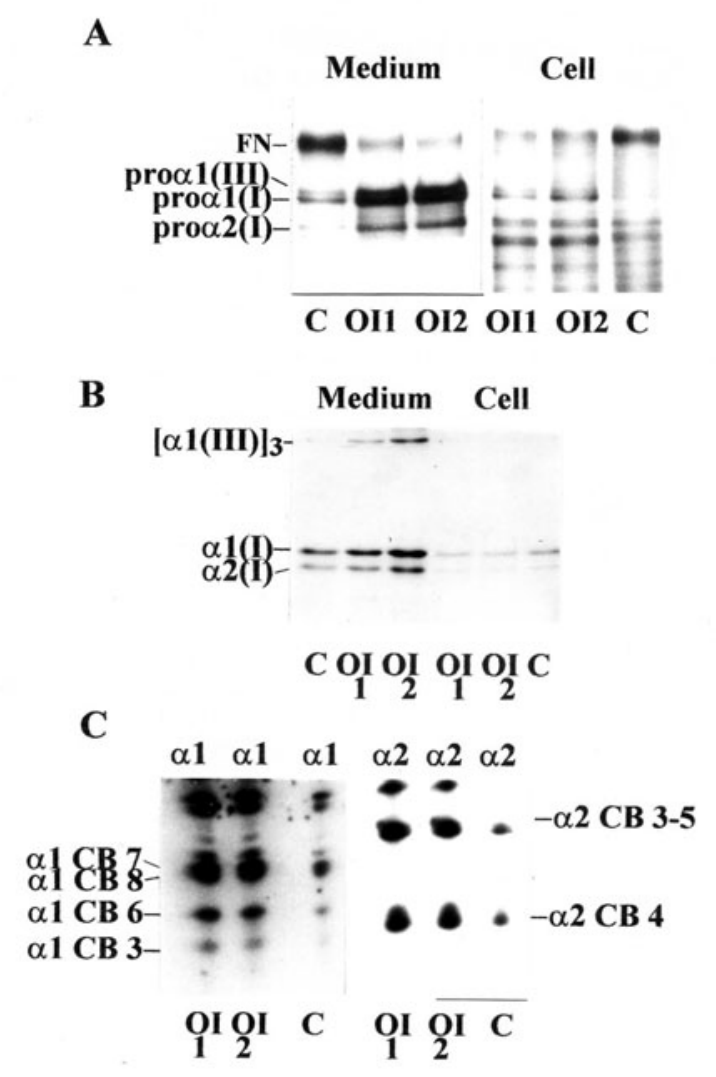

Figure 2. SDS/PAGE of procollagen (A) collagen (B) and $\mathrm{CNBr}$ peptides (C).

For (A), (B) and (C) OI1 and OI2 represent twins, C control. Fibroblasts were incubated in ascorbate-supplemented serum-free medium with $\left[{ }^{3} \mathrm{H}\right]$ proline for $18 \mathrm{~h}$. Procollagen from medium and cell layers was analyzed on $5 \%$ gel under reducing conditions (A). The migration positions of type I and III procollagen chains, and fibronectin (FN) are shown. Pepsin-digested collagen was electrophoresed on 5\% gel under nonreducing conditions (B). Gel strips corresponding to the position of type I collagen chains (B) were excised and incubated for $2 \mathrm{~h}$ in 5\% $\mathrm{CNBr}$ and 70\% formic acid, and separated in the second dimension on $12.5 \%$ gel (C). 
$\mathbf{A}$

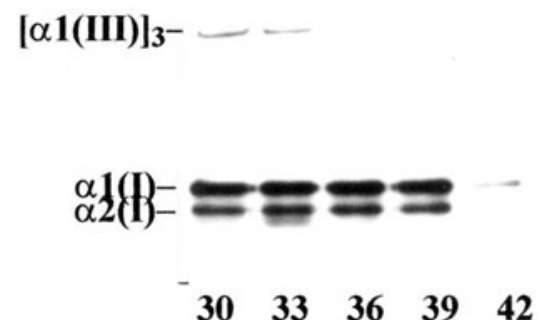

B
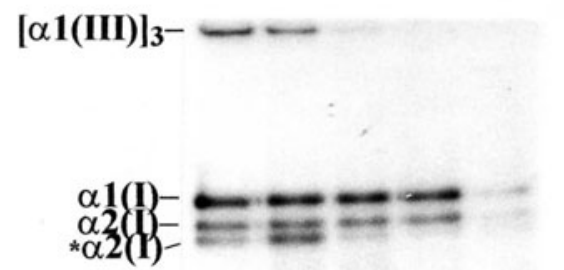

$\begin{array}{lllll}30 & 33 & 36 & 39 & 42\end{array}$

C

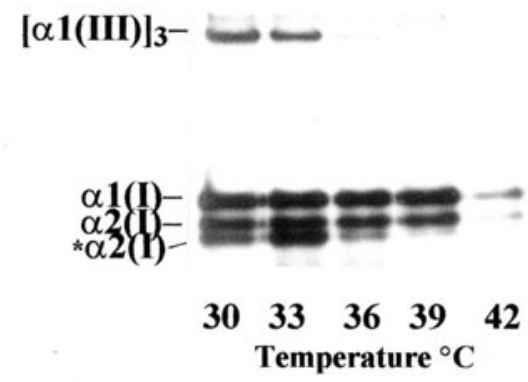

Figure 3. Enzymatic digestion of control (A), OI1 (B) and OI2 (C) media collagens.

$\left[{ }^{3} \mathrm{H}\right]$ proline labeled collagen was digested with trypsin for 5 min at increasing temperatures. Type I and III collagen as well as shortened ${ }^{*} \alpha 2(\mathrm{I})$ chains are shown. The thermal stability $t_{m}$ for the truncated ${ }^{*} \alpha$ 2(I) chain $\left(36^{\circ} \mathrm{C}\right)$ was decreased by $6^{\circ} \mathrm{C}$ as compared with normal chains.

N-terminal portion of the $\alpha 2$ (I) chain. The corresponding mRNA region of $\alpha 2(\mathrm{I})$ collagen (579 bp) was amplified by RT-PCR and asymmetric PCR. Direct sequencing of the asymmetric PCR product was performed. The sequence corresponding to the first nucleotide of the codon for amino acid 92 contained two bands of equal intensity co-migrating in the $\mathrm{C}$ and $\mathrm{G}$ reaction lanes (Fig. 4). The CAC codon corresponds to the normal His at that position, while the GAC codon re- sults in an aspartic acid substitution for the histidine. The same data were obtained for OI1 and OI2 indicating that both probands were heterozygous for the codon of amino acid 92 of $\alpha 2$ (I) procollagen, with one allele coding for histidine and the other for aspartic acid. No other sequence changes were identified in the amino end of the helical part of $\alpha 2(\mathrm{I})$ chain.

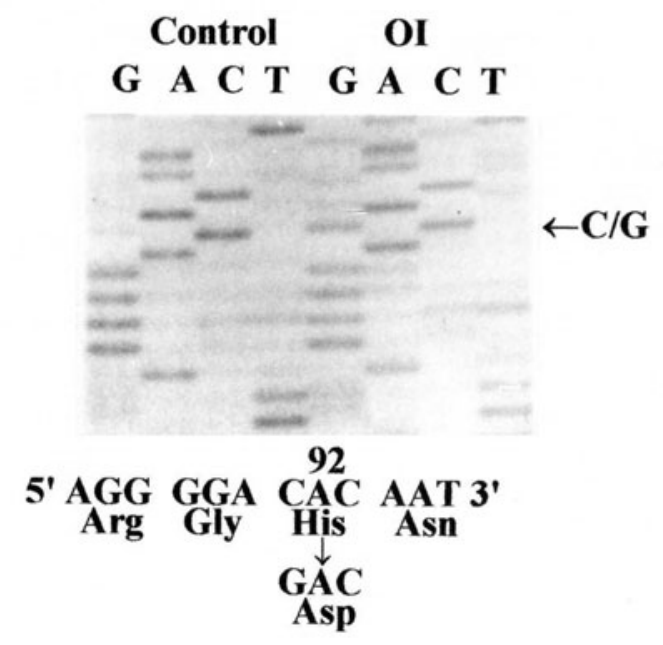

Figure 4. DNA sequence of single-stranded PCR product obtained by asymmetric amplification of control and OI1 $\alpha 2(\mathrm{I})$ cDNA.

Dideoxy sequencing of cDNA reveals the 405C > G mutation, predicting a histidine to aspartic acid substitution at position 92 in the $\alpha 2(\mathrm{I})$ chain. The same His92Asp substitution was detected in OI2 $\alpha 2$ (I) cDNA (not shown).

Previous studies on the processing of extracellular type I procollagen established that even small changes in the primary structure of procollagen can either make the protein totally resistant to cleavage by N-proteinase or alter the rate of its processing (Tuderman \& Prockop, 1982). We have examined the rate of procollagen processing by analyzing the appearance of its intermediate $\mathrm{pC}$ forms. During processing of secreted type I procollagen to collagen by pericellular peptidases, the $\mathrm{pC}-\alpha 1$ (I) form appeared earlier in control cultured fibroblasts (Fig. 5) suggesting a slower rate of processing in OI fibroblasts. 


\section{DISCUSSION}

In most instances the perinatal lethal form of OI results from substitutions for single glycyl residues within the triple helical domain of $\alpha 1$ (I) or $\alpha 2(\mathrm{I})$ collagen chains (Kuivaniemi et al., 1991; Prockop, 1992). The requirement for glycine at every third position in the triple helical domain is known and confirmed by consequences of many glycyl substitutions found in osteogenesis imperfecta. These mutations interfere with the formation of stable triple helix, delay secretion of mole-

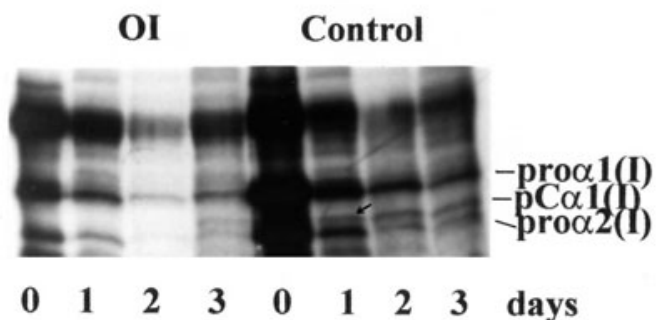

Figure 5. Pericellular processing of type I procollagen synthesized by $\mathrm{OI} 1$ and normal cultured fibroblasts.

Confluent cells were labeled with $\left[{ }^{3} \mathrm{H}\right]$ proline for $24 \mathrm{~h}$ and then chased with nonradioactive proline. Media were harvested from independent wells of fibroblasts at 24-h intervals over a 3-day period. Procollagen was precipitated from the media and electrophoresed on 5\% polyacrylamide/urea/SDS gel with DTT. Arrow indicates the $\mathrm{pC}-\alpha 1$ (I) form (after cleaving of the N-terminal peptide) appearing earlier in the control sample. The same rate of processing was observed in OI2 fibroblasts (not shown).

cules that contain mutant chains, alter the extent of posttranslational modification of all chains in mutated molecules, interfere with amino-terminal propeptide cleavage, and result in an abnormal structure of type I procollagen molecules that may affect fibrillogenesis (Byers, 1993; Cole, 1997; Bachinger et al., 1993). However, there is little information regarding the function of non-glycine residues in the triple helical domain. In this study we report an amino acid substitution at a non-glycine residue in the
N-terminal end of the triple-helical domain of type I collagen. This single base substitution converts histidine 92 at the $\mathrm{X}$ position of a Gly-X-Y repeat to aspartic acid. The histidine side chain is hydrophobic and rigid, whereas the side chain of aspartic acid is charged and more flexible. His89 in the $\alpha 1$ (I) chain seems to be an important residue (Kuznetsova et al., 2001). It is located within the Gly-X-HylGly-His-Arg-Gly sequence that has been suspected as a recognition site that drives fiber assembly (Veis \& Payne, 1988). It is the largest conserved fragment in all fibril-forming collagens repeated near each end of each polypeptide chain. In addition, adjacent hydroxylysine at position 87 is a well-known crosslinking site (Veis \& Payne, 1988). His92 in the $\alpha 2$ (I) chain seems to be the $\alpha 2$ counterpart of $\alpha 1$ (I) His89.

The collagen molecules with the His92Asp substitution showed increased susceptibility to protease digestion. Trypsin cleaved about $50 \%$ of the $\alpha 2$ (I) chains but not $\alpha 1$ (I) molecules and the cleaved fragment was stable to trypsin digestion at temperatures up to $33^{\circ} \mathrm{C}$. The trimers with the detected substitution accounted for $50 \%$ of the total trimers and thus they were all digested by the enzyme. Although the cleavage site of the $\alpha 2$ chain was not determined, the size of the shortened collagen band estimated by comparing its electrophoretic migration with the $\alpha 1$ (I) and $\alpha 2$ (I) collagen chains suggests that the cleavage was close to the substitution. There is a possibility that this substitution or some other structural defects can introduce a conformational change that exposes residues normally inaccessible to the proteolytic enzyme. However, the marked reduction in the thermal stability of the collagen molecule containing shortened $\alpha 2$ chains is striking. Batge et al. (1990) purified from human bones a truncated form of type I collagen containing shortened $\alpha 1$ chains at Leu95 with decreased thermal stability $\left(t_{\mathrm{m}}\right.$ was $\left.36.5^{\circ} \mathrm{C}\right)$. They assumed that there might be a rigid region among residues 1-95 stabilizing the triple helix. It was noted 
that the most severe clinical phenotypes are caused by mutations occurring in imino acid rich domains (Bachinger et al., 1993). However, the most stabilizing tripeptide sequence is Gly-Pro-Hyp and it is repeated only once within the first 95 residues of the $\alpha 2$ and three times in $\alpha 1$ chains. The highly destabilizing effect on the collagen thermal stability of glycine substitutions is well known (Beck et al., 2000). The His92Asp substitution identified in this work does not exclude the possibility of some other alterations in the primary structure affecting the thermal stability of collagen.

The His92Asp mutation, however, did not have any major effect on the biosynthesis or secretion of procollagen. There was no evidence of posttranslational overmodification of pro $\alpha$ chains; the ratio of normal to shortened pro $\alpha 2(\mathrm{I})$ chains in the medium after trypsin digestion was about 1:1. However, the presence of this alteration could influence the processing of procollagen. The type I procollagen N-proteinase is unusual among proteinases in that it requires a procollagen substrate with a native conformation and that even small changes in the primary structure of type I procollagen can alter the rate of its processing (Tuderman \& Prockop, 1982). Mutations which produce incomplete cleavage of the N-propeptides have previously been detected in patients with type VII of EhlersDanlos syndrome either because of an undefined structural alterations of the pro $\alpha 1$ (I) or pro $\alpha 2$ (I) chains or procollagen N-proteinase (Colige et al., 1999). Although the reported non-glycine substitution by itself can not account for the lethal phenotype, it may play some role in the disorder in combination with other factors.

\section{R E F E R E N C E S}

Bachinger HP, Morris NP, Davis JM. (1993) Thermal stability and folding of the collagen triple-helix and the effects of mutations in osteogenesis imperfecta on the triple-helix of type I collagen. Am J Med Genet.; 45: 152-62.

Batge B, Notbohm H, Diebold J, Lehmann H, Bodo M, Deutzmann R, Muller PK. (1990) A critical crosslink region in human-bone-derived collagen type I. Specific cleavage site at residue Leu95. Eur J Biochem.; 192: 153-9.

Beck K, Chan VC, Shenoy N, Kirkpatrick A, Ramshaw JAM, Brodsky B. (2000) Destabilization of osteogenesis imperfecta collagen-like model peptides correlates with the identity of the residue replacing glycine. Proc Natl Acad Sci U S A.; 93: 4273-8.

Berg RA, Prockop DJ. (1973) The thermal transition of a non-hydroxylated form of collagen. Evidence for a role for hydroxyproline in stabilizing the triple-helix of collagen. Biochem Biophys Res Commun.; 52: 115-20.

Bonadio J, Holbrook KA, Gelinas RE, Jacob J, Byers PH. (1985) Altered triple helical structure of type I procollagen in lethal perinatal osteogenesis imperfecta. J Biol Chem.; 260: 1734-42.

Byers PH. (1993) Osteogenesis imperfecta. In Connective tissue and its heritable disorders: molecular, genetic and medical aspects. Royce PM, Steinmann B, eds, pp 317-50. Wiley-Liss, New York.

Byers PH. (2001) Folding defects in fibrillar collagens. Philos Trans R Soc London, Ser B.; 356: 151-8.

Cole WG. (1997) The molecular pathology of osteogenesis imperfecta. Clin Orthop.; 343: $235-48$.

Colige A, Sieron AL, Li SW, Schwarze U, Petty E, Wertelecki W, Wilcox W, Krakow D, Cohn DH, Reardon W, Byers PH, Lapiere CM, Prockop DJ, Nusgens BV. (1999) Human Ehlers-Danlos syndrome type VII C and bovine dermatosparaxis are caused by mutations in the procollagen I N-proteinase gene. Am J Hum Genet.; 65: 308-17.

Gajko-Galicka A. (2002) Mutations in type I collagen genes resulting in osteogenesis imperfecta in humans. Acta Biochim Polon.; 49: 433-41. 
Galicka A, Wolczynski S, Lesniewicz R, Chyczewski L, Gindzienski A. (2002) A novel Gly to Arg substitution at position 388 of the 1 chain of type I collagen in lethal form of osteogenesis imperfecta. Acta Biochim Polon.; 49: $443-50$.

Kuivaniemi H, Tromp G, Prockop DJ. (1991) Mutations in collagen genes: causes of rare and some common diseases in humans. FASEB J.; 5: 2052-60.

Kuznetsova N, McBride DJ Jr, Leikin S. (2001) Osteogenesis imperfecta murine: interactions between type I collagen homotrimers. $J \mathrm{Mol}$ Biol.; 309: 807-15.

Laemmli UK. (1970) Cleavage of structural proteins during the assembly of the head of bacteriophage T4. Nature.; 227: 680-5.

McLaughlin SH, Bulleid NJ. (1998) Molecular recognition in procollagen chain assembly. Matrix Biol.; 16: 369-77.
Peterkofsky B, Chojkier M, Bateman J. (1982) Determination of collagen synthesis in tissue and cell culture system. In Immunochemistry of the extracellular matrix. Furthmay $\mathrm{M}$, ed, pp 19-47. CRC Press, Boca Raton, Fl.

Prockop DJ. (1992) Mutations in collagen genes as a cause of connective-tissue diseases. $N$ Engl J Med.; 326: 540-6.

Sanger F, Nicklin S, Coulson AR. (1997) DNA sequencing with chain terminating inhibitors. Proc Natl Acad Sci U S A.; 74: 5463-7.

Sillence DO, Senn A, Danks DM. (1979) Genetic heterogeneity in osteogenesis imperfecta. $J$ Med Genet.; 16: 101-16.

Tuderman L, Prockop DJ. (1982) Procollagen N-proteinase. Properties of the enzyme purified from chick embryo tendons. Eur $J$ Biochem.; 125: 545-9.

Veis A, Payne K. (1988) Collagen fibrillogenesis. In Collagen. Nimni ME, ed, vol 1, pp 113-37. CRC Press, Boca Raton, Fl. 